Moroccan J. of Pure and Appl. Anal. (MJPAA)

Volume 7(3), 2021, Pages 400-412

ISSN: Online 2351-8227 - Print 2605-6364

DOI: $10.2478 / \mathrm{mjpaa}-2021-0027$

\title{
On a Class of Caputo Time Fractional Problems with Boundary Integral Conditions
}

\author{
Karim Aggoun ${ }^{1}$ and Ahcene Merad ${ }^{2}$
}

Aвstract. The aim of this paper is to work out the solvability of a class of Caputo time fractional problems with boundary integral conditions. A generalized formula of integration is demonstrated and applied to establish the a priori estimate of the solution, then we prove the existence which is based on the range density of the operator associated with the problem.

Mathematics Subject Classification (2020). 35R11, 35D35.

Key words and phrases. Time fractional problem, A priori estimate, Boundary integral conditions.

\section{Introduction}

Let $Q$ be a rectangle defined by $Q=(0,1) \times(0, T)$ and considering the fractional equation

$$
\partial_{0 t}^{\alpha} u+(-1)^{m} \frac{\partial^{m}}{\partial x^{m}}\left(a(x, t) \frac{\partial^{m} u}{\partial x^{m}}\right)=f(x, t)
$$

where $m \geq 1$ and $\partial_{0 t}^{\alpha}$ denotes the Caputo time fractional derivative of order $0<\alpha<1$ with lower bound 0 , subject to the initial condition

$$
u(x, 0)=\varphi(x), \quad x \in(0,1)
$$

Received : November 14, 2020 - Accepted: March 31, 2021.

(C) The Author(s) 2021. This article is published with open access by Sidi Mohamed Ben Abdallah University. 1,2Department of Mathematics,Laboratory of Dynamical Systems and Control, University Larbi Ben M'hidi, Oum El Bouaghi, Algeria.

e-mail ${ }^{1}$ : aggoun.karim@gmail.com (Corresponding Author)

e-mail ${ }^{2}$ : merad_ahcene@yahoo.fr, merad.ahcene@univ-oeb.dz. 
and the boundary integral conditions

$$
\int_{0}^{1} x^{k} u(x, t) d x=0, \quad k=\overline{0,2 m-1} .
$$

Many instances of problems described by equation (1.1) have been investigated. For example the case $m=1$ in [1] and [8]. Other authors studied the integer order case ([2], [4] and [5]). We started by studying the time fractional problem in the Caputo sense applying the energy inequality method which is a powerful functional analysis method for showing problems wellposedness, it is mainly based on an a priori estimate to prove uniqueness of the strong solution, meanwhile the range density of the operator generated by the problem ensures its existence. This method has been approached by several authors for many integer order problems with classical, non-local and mixed conditions, but only few fractional problems have been studied.

In this paper we start section 2 with some preliminaries by giving some definitions, function spaces and other key tools, then we establish a generalized formula of integration by parts applicable to the class of problems with integral conditions (1.3). In section 3, we choose convenient spaces and multiplier to ascertain the a priori estimate that will be extended to conclude the uniqueness of the strong solution and its dependence on the data given in problem (1.1)(1.3). Finally, we prove the existence in section 4 relying on the fact that the operator generated by our problem is dense, then we present an example in which we illustrate the usefulness of the results obtained in the previous sections.

\section{Preliminaries}

These definitions and tools are necessary:

- The Caputo fractional derivative $\partial_{0 t}^{\alpha}$ is defined for a differentiable function $v$ by

$$
\partial_{0 t}^{\alpha} v(t)=I^{1-\alpha} v^{\prime}(t)=\frac{1}{\Gamma(1-\alpha)} \int_{0}^{t} \frac{v^{\prime}(\tau)}{(t-\tau)^{\alpha}} d \tau, \quad t>0,
$$

where $\Gamma$ is the gamma function and $I^{\alpha}$ is the Riemann-Liouville integral operator defined for $0<\alpha<1$ by

$$
I^{\alpha} v(t)=\frac{1}{\Gamma(\alpha)} \int_{0}^{t} \frac{v(\tau)}{(t-\tau)^{1-\alpha}} d \tau .
$$

Another formula of the Caputo fractional derivative can be obtained in our case $(1-\alpha>0)$ is

$$
\begin{aligned}
I^{1-\alpha} v^{\prime}(t) & =\frac{d}{d t} I^{2-\alpha} v^{\prime}(t) \\
& =\frac{1}{\Gamma(1-\alpha)} \frac{d}{d t} \int_{0}^{t} \frac{v(\tau)-v(0)}{(t-\tau)^{\alpha}} d \tau .
\end{aligned}
$$


for more about fractional calculus see [7].

- $C^{k}(\bar{\Omega})$ denotes the space of $k$-fold differentiable functions on $\bar{\Omega}$.

- $L^{2}(0, T)$ is the space of measurable square-integrable functions on $(0, T)$.

- $C_{0}(0,1)$ is the space of continuous functions with compact support in $(0,1)$. We define on $C_{0}(0,1)$ the bilinear form given by

$$
((u, v))=\int_{0}^{1} \Im_{x}^{k} u \Im_{x}^{k} v d x,
$$

where $\Im_{x}^{0} u=u$ and

$$
\Im_{x}^{k} u(x, t)=\frac{1}{(k-1) !} \int_{0}^{x} \frac{u(\xi, t)}{(x-\xi)^{1-k}} d \xi, \quad k \geq 1 .
$$

This bilinear form is considered as a scalar product on $C_{0}(0,1)$ but is not complete. Therefore we define :

- The Bouziani space $B_{2}^{k}(0,1)$ as a completion of the space $C_{0}(0,1)$ for the scalar product (2.3), the associated norm is

$$
\begin{aligned}
\|u\|_{B_{2}^{k}(0,1)} & =\sqrt{((u, u))} \\
& =\left\|\Im_{x}^{k} u\right\|_{L^{2}(0,1)} .
\end{aligned}
$$

Corollary 2.1. For $k \in \mathbb{N}$ we have

$$
\|u\|_{B_{2}^{k}(0,1)}^{2} \leq \frac{1}{2^{k}}\|u\|_{L^{2}(0,1)}^{2} .
$$

Proof. See corollary of lemma 1 in [2] for $b=1$.

Lemma 2.1. for any absolutely continuous function $v(t)$ on the interval $(0, T)$, we have the inequality

$$
2 v(t) \partial_{0 t}^{\alpha} v(t) \geq \partial_{0 t}^{\alpha}(v(t))^{2}, \quad 0<\alpha<1 .
$$

Proof. See lemma 1 in [1].

Lemma 2.2. Let a non-negative absolutely continuous function $y(t)$ satisfy the inequality

$$
\partial_{0 t}^{\alpha} y(t) \leq c_{1} y(t)+c_{2}(t), \quad 0<\alpha<1,
$$

for almost all $t$ in $[0, T]$, where $c_{1}>0$ and $c_{2}(t)$ is an integrable non-negative function on $[0, T]$. Then

$$
y(t) \leq y(0) E_{\alpha}\left(c_{1} t^{\alpha}\right)+\Gamma(\alpha) E_{\alpha, \alpha}\left(c_{1} t^{\alpha}\right) I^{\alpha} c_{2}(t) .
$$

where

$$
\begin{gathered}
E_{\alpha}(z)=\sum_{k=0}^{\infty} \frac{z^{k}}{\Gamma(\alpha k+1)}, \\
E_{\alpha, \beta}(z)=\sum_{k=0}^{\infty} \frac{z^{k}}{\Gamma(\alpha k+\beta)}
\end{gathered}
$$

are the Mittag-leffler functions. 
Proof. See Lemma 2 in [1].

- Cauchy inequality with $\varepsilon$

$$
2 A B \leq \varepsilon A^{2}+\frac{1}{\varepsilon} B^{2}
$$

where $A$ and $B$ are real numbers.

- Vandermonde identity

$$
\sum_{i=0}^{k}\left(\begin{array}{c}
m-1 \\
2 k-1-i
\end{array}\right)\left(\begin{array}{l}
k \\
i
\end{array}\right)=\left(\begin{array}{c}
m+k-1 \\
2 k-1
\end{array}\right)
$$

where $m$ and $k$ are positive integers.

- We need the coefficient $K_{n}^{k}$ defined for all $(k, n) \in \mathbb{N}^{2}$ by

$$
\begin{cases}K_{n}^{k}=0, & k>n \\ K_{0}^{0}=2, & k \geq 1 \\ K_{k}^{0}=0, & k \geq 1 \\ K_{k}^{k}=(-1)^{k}, & 1 \leq k \leq n \\ K_{n}^{k}=-\left(K_{n-1}^{k-1}+K_{n-2}^{k-1}\right),\end{cases}
$$

Proposition 2.1. For all $(k, n) \in \mathbb{N}^{2}$ we have

$$
\left\{\begin{array}{ll}
K_{0}^{0}=2, & \\
K_{k}^{0}=0, & k \geq 1 \\
K_{n}^{k}=(-1)^{k} \frac{n}{k}\left(\begin{array}{c}
k \\
n-k
\end{array}\right) & k, n \geq 1
\end{array} .\right.
$$

Proof. We show that the last equation of (2.7) is satisfied, by reducing to the common denominator.

- We also introduce a similar coefficient $A_{m}^{k}$ defined for all $(m, k) \in \mathbb{N}^{2}$

$$
\begin{cases}A_{m}^{0}=2, & m \geq 0 \\
A_{m}^{k}=(-1)^{k} \frac{m}{k}\left(\begin{array}{c}
m+k-1 \\
2 k-1
\end{array}\right), & k>0\end{cases}
$$

Proposition 2.2. For all $(m, k) \in \mathbb{N}^{2}$

$$
A_{m}^{k}=\sum_{n=k}^{\min \{m, 2 k\}}\left(\begin{array}{l}
m \\
n
\end{array}\right) K_{n}^{k} .
$$

Proof. It is sufficient to prove that

$$
\sum_{n=k}^{2 k}\left(\begin{array}{c}
m-1 \\
n-1
\end{array}\right)\left(\begin{array}{c}
k \\
n-k
\end{array}\right)=\left(\begin{array}{c}
m+k-1 \\
2 k-1
\end{array}\right)
$$

We have

$$
\sum_{n=k}^{2 k}\left(\begin{array}{c}
m-1 \\
n-1
\end{array}\right)\left(\begin{array}{c}
k \\
n-k
\end{array}\right)=\sum_{n=k}^{2 k}\left(\begin{array}{c}
m-1 \\
n-1
\end{array}\right)\left(\begin{array}{c}
k \\
2 k-n
\end{array}\right)
$$




$$
=\sum_{i=0}^{k}\left(\begin{array}{c}
m-1 \\
2 k-1-i
\end{array}\right)\left(\begin{array}{l}
k \\
i
\end{array}\right)
$$

where Vandermonde identity (2.6) ends the proof.

These numbers $\left(K_{n}^{k}\right.$ and $\left.A_{m}^{k}\right)$ and their relation given in proposition 2.2 are crucial for the next proposition.

Proposition 2.3. (Generalized formula of integration)

Let a function $u$ satisfies integral conditions (1.3) and

$$
u \in B_{2}^{k}(0,1), \frac{\partial^{k} u}{\partial x^{k}} \in L^{2}(0,1), \quad k=\overline{0, m},
$$

then for all $a(\cdot, t) \in C^{2 m}([0,1])$ we have

$$
2(-1)^{m} \int_{0}^{1} a \frac{\partial^{m} u}{\partial x^{m}} \Im_{x}^{m} u d x=\sum_{k=0}^{m} A_{m}^{k} \int_{0}^{1} \frac{\partial^{2 k} a}{\partial x^{2 k}}\left(\Im_{x}^{k} u\right)^{2} d x
$$

Proof. Let $u$ verifies (2.13) and $a(\cdot, t) \in C^{2 m}([0,1])$, we first show by induction on $k$ that the integration by parts $k$ times $(0 \leq k \leq m)$ using integral conditions (1.3) gives

$$
\int_{0}^{1} a \frac{\partial^{m} u}{\partial x^{m}} \Im_{x}^{m} u d x=(-1)^{k} \sum_{n=0}^{k}\left(\begin{array}{l}
k \\
n
\end{array}\right) \int_{0}^{1} \frac{\partial^{n} a}{\partial x^{n}} \frac{\partial^{m-k} u}{\partial x^{m-k}} \Im_{x}^{m+n-k} u d x,
$$

indeed a simple integration by parts of the right-hand side using condition (1.3) leads to

$$
\begin{aligned}
\int_{0}^{1} a \frac{\partial^{m} u}{\partial x^{m}} \Im_{x}^{m} u d x & =(-1)^{k+1} \sum_{n=0}^{k}\left(\begin{array}{l}
k \\
n
\end{array}\right) \int_{0}^{1} \frac{\partial^{n+1} a}{\partial x^{n+1}} \frac{\partial^{m-(k+1)} u}{\partial x^{m-(k+1)}} \Im_{x}^{m+n-k} u d x \\
& +(-1)^{k+1} \sum_{n=0}^{k}\left(\begin{array}{l}
k \\
n
\end{array}\right) \int_{0}^{1} \frac{\partial^{n} a}{\partial x^{n}} \frac{\partial^{m-(k+1)} u}{\partial x^{m-(k+1)}} \Im_{x}^{m+n-(k+1)} u d x,
\end{aligned}
$$

if we replace $n$ by $n-1$ in the first term of the right-hand side of the above equality we get

$$
\begin{aligned}
\int_{0}^{1} a \frac{\partial^{m} u}{\partial x^{m}} \Im_{x}^{m} u d x & =(-1)^{k+1} \sum_{n=1}^{k+1}\left(\begin{array}{c}
k \\
n-1
\end{array}\right) \int_{0}^{1} \frac{\partial^{n} a}{\partial x^{n}} \frac{\partial^{m-(k+1)} u}{\partial x^{m-(k+1)}} \Im_{x}^{m+n-(k+1)} u d x \\
& +(-1)^{k+1} \sum_{n=0}^{k}\left(\begin{array}{l}
k \\
n
\end{array}\right) \int_{0}^{1} \frac{\partial^{n} a}{\partial x^{n}} \frac{\partial^{m-(k+1)} u}{\partial x^{m-(k+1)}} \Im_{x}^{m+n-(k+1)} u d x
\end{aligned}
$$

Hence,

$$
\int_{0}^{1} a \frac{\partial^{m} u}{\partial x^{m}} \Im_{x}^{m} u d x=
$$




$$
\begin{gathered}
(-1)^{k+1} \sum_{n=1}^{k}\left\{\left(\begin{array}{l}
k \\
n
\end{array}\right)+\left(\begin{array}{c}
k \\
n-1
\end{array}\right)\right\} \int_{0}^{1} \frac{\partial^{n} a}{\partial x^{n}} \frac{\partial^{m-(k+1)} u}{\partial x^{m-(k+1)}} \Im_{x}^{m+n-(k+1)} u d x \\
+(-1)^{k+1}\left(\int_{0}^{1} a \frac{\partial^{m-(k+1)} u}{\partial x^{m-(k+1)}} \Im_{x}^{m-(k+1)} u d x+\int_{0}^{1} \frac{\partial^{k+1} a}{\partial x^{k+1}} \frac{\partial^{m-(k+1)} u}{\partial x^{m-(k+1)}} \Im_{x}^{m} u d x\right),
\end{gathered}
$$

knowing that

$$
\begin{aligned}
\left(\begin{array}{c}
k+1 \\
0
\end{array}\right) & =\left(\begin{array}{l}
k+1 \\
k+1
\end{array}\right) \\
& =1
\end{aligned}
$$

and

equation (2.17) becomes

$$
\left(\begin{array}{l}
k \\
n
\end{array}\right)+\left(\begin{array}{c}
k \\
n-1
\end{array}\right)=\left(\begin{array}{c}
k+1 \\
n
\end{array}\right)
$$

$$
\int_{0}^{1} a \frac{\partial^{m} u}{\partial x^{m}} \Im_{x}^{m} u d x=(-1)^{k+1} \sum_{n=0}^{k+1}\left(\begin{array}{c}
k+1 \\
n
\end{array}\right) \int_{0}^{1} \frac{\partial^{n} a}{\partial x^{n}} \frac{\partial^{m-(k+1)} u}{\partial x^{m-(k+1)}} \Im_{x}^{m+n-(k+1)} u d x,
$$

and the identity (2.14) is proved. The particular case of identity (2.14)

$$
2(-1)^{m} \int_{0}^{1} a \frac{\partial^{m} u}{\partial x^{m}} \Im_{x}^{m} u d x=2 \sum_{n=0}^{m}\left(\begin{array}{c}
m \\
n
\end{array}\right) \int_{0}^{1} \frac{\partial^{n} a}{\partial x^{n}} u \Im_{x}^{n} u d x
$$

is needed. Secondly we prove by induction on $n(n<m)$ that

$$
2 \int_{0}^{1} \frac{\partial^{n} a}{\partial x^{n}} u \Im_{x}^{n} u d x=\sum_{k=\left\lceil\frac{n}{2}\right\rceil}^{n} K_{n}^{k} \int_{0}^{1} \frac{\partial^{2 k} a}{\partial x^{2 k}}\left(\Im_{x}^{k} u\right)^{2} d x
$$

Assume equality (2.20) holds for $n$ and $n-1$, by noticing that $0 \leq k<\left\lceil\frac{n}{2}\right\rceil \Rightarrow K_{n}^{k}=0$, we can write

$$
2 \int_{0}^{1} \frac{\partial^{n} a}{\partial x^{n}} u \Im_{x}^{n} u d x=\sum_{k=0}^{n} K_{n}^{k} \int_{0}^{1} \frac{\partial^{2 k} a}{\partial x^{2 k}}\left(\Im_{x}^{k} u\right)^{2} d x
$$

and

$$
2 \int_{0}^{1} \frac{\partial^{n-1} a}{\partial x^{n-1}} u \Im_{x}^{n-1} u d x=\sum_{k=0}^{n-1} K_{n-1}^{k} \int_{0}^{1} \frac{\partial^{2 k} a}{\partial x^{2 k}}\left(\Im_{x}^{k} u\right)^{2} d x
$$

Replacing $u$ by $\Im_{x} u$ and $a$ by $\frac{\partial^{2} a}{\partial x^{2}}$ in equations (2.21) and (2.22) yields

$$
2 \int_{0}^{1} \frac{\partial^{n+2} a}{\partial x^{n+2}} \Im_{x} u \Im_{x}^{n+1} u d x=\sum_{k=0}^{n} K_{n}^{k} \int_{0}^{1} \frac{\partial^{2(k+1)} a}{\partial x^{2(k+1)}}\left(\Im_{x}^{k+1} u\right)^{2} d x
$$


and

$$
2 \int_{0}^{1} \frac{\partial^{n+1} a}{\partial x^{n+1}} \Im_{x} u \Im_{x}^{n} u d x=\sum_{k=0}^{n-1} K_{n-1}^{k} \int_{0}^{1} \frac{\partial^{2(k+1)} a}{\partial x^{2(k+1)}}\left(\Im_{x}^{k+1} u\right)^{2} d x
$$

A simple integration by parts using condition (1.3) gives

$$
2 \int_{0}^{1} \frac{\partial^{n+1} a}{\partial x^{n+1}} u \Im_{x}^{n+1} u d x=-2 \int_{0}^{1} \frac{\partial^{n+2} a}{\partial x^{n+2}} \Im_{x} u \Im_{x}^{n+1} u d x-2 \int_{0}^{1} \frac{\partial^{n+1} a}{\partial x^{n+1}} \Im_{x} u \Im_{x}^{n} u d x
$$

and the substitution of equations (2.23) and (2.24) in (2.25) leads to

$$
\begin{aligned}
2 \int_{0}^{1} \frac{\partial^{n+1} a}{\partial x^{n+1}} u \Im_{x}^{n+1} u d x & =-\sum_{k=0}^{n-1}\left(K_{n}^{k}+K_{n-1}^{k}\right) \int_{0}^{1} \frac{\partial^{2(k+1)} a}{\partial x^{2(k+1)}}\left(\Im_{x}^{k+1} u\right)^{2} d x \\
& -K_{n}^{n} \int_{0}^{1} \frac{\partial^{2(n+1)} a}{\partial x^{2(n+1)}}\left(\Im_{x}^{n+1} u\right)^{2} d x
\end{aligned}
$$

notice that $-K_{n}^{n}=K_{n+1}^{n+1}$ and from the recurrence formula in (2.7) we have $-\left(K_{n}^{k}+K_{n-1}^{k}\right)=$ $K_{n+1}^{k+1}$, then replacing $k$ by $k-1$ in the above equation gives

$$
2 \int_{0}^{1} \frac{\partial^{n+1} a}{\partial x^{n+1}} u \Im_{x}^{n+1} u d x=\sum_{k=0}^{n+1} K_{n+1}^{k} \int_{0}^{1} \frac{\partial^{2 k} a}{\partial x^{2 k}}\left(\Im_{x}^{k} u\right)^{2} d x
$$

since $K_{n+1}^{0}=0$. This proves identity (2.20). Finally, we substitute equation (2.20) in (2.19) to get

$$
2(-1)^{m} \int_{0}^{1} a \frac{\partial^{m} u}{\partial x^{m}} \Im_{x}^{m} u d x=\sum_{n=0}^{m}\left(\begin{array}{c}
m \\
n
\end{array}\right) \sum_{k=\left\lceil\frac{n}{2}\right\rceil}^{n} K_{n}^{k} \int_{0}^{1} \frac{\partial^{2 k} a}{\partial x^{2 k}}\left(\Im_{x}^{k} u\right)^{2} d x
$$

then by rearranging the sums we obtain

$$
2(-1)^{m} \int_{0}^{1} a \frac{\partial^{m} u}{\partial x^{m}} \Im_{x}^{m} u d x=\sum_{k=0}^{m} \sum_{n=k}^{\min \{m, 2 k\}}\left(\begin{array}{c}
m \\
n
\end{array}\right) K_{n}^{k} \int_{0}^{1} \frac{\partial^{2 k} a}{\partial x^{2 k}}\left(\Im_{x}^{k} u\right)^{2} d x
$$

where proposition 2.2 finishes the proof.

\section{A priori estimate and consequences}

To establish the existence and uniqueness of the solution of problem (1.1)-(1.3) we write it in an equivalent operator form so that it can be viewed as the solution of this operator equation

$$
\mathscr{L} u=\mathcal{F}
$$


with $\mathscr{L}=(\mathcal{L}, \ell)$ acts from $B$ to $H$ where

$$
\begin{gathered}
B=\left\{u \in L^{2}\left(B_{2}^{k}(0,1),(0, T)\right), k=\overline{0, m} / \frac{\partial^{k} u}{\partial x^{k}} \in L^{2}(Q), k=\overline{0, m},\right. \\
\left.\partial_{0 t}^{\alpha} u \in L^{2}\left(B_{2}^{m}(0,1),(0, T)\right)\right\}
\end{gathered}
$$

is the Banach space of functions $u$ endowed by the finite norm

$$
\|u\|_{B}^{2}=\sup _{0 \leq t \leq T} I^{1-\alpha}\|u\|_{B_{2}^{m}(0,1)}^{2}+\sum_{k=0}^{m-1}\|u\|_{L^{2}\left(B_{2}^{k}(0,1),(0, T)\right)}^{2},
$$

$H$ is the Hilbert space consisting of vector-valued functions $\mathcal{F}=(f, \varphi)$ with finite norm

$$
\|\mathcal{F}\|_{H}^{2}=\|f\|_{L^{2}(Q)}^{2}+\|\varphi\|_{L^{2}(0,1)}^{2},
$$

and

$$
\left\{\begin{array}{l}
\mathcal{L} u=\partial_{0 t}^{\alpha} u+(-1)^{m} \frac{\partial^{m}}{\partial x^{m}}\left(a(x, t) \frac{\partial^{m} u}{\partial x^{m}}\right) \\
\ell u=u(x, 0)
\end{array} .\right.
$$

The domain of definition of the operator $\mathscr{L}$ is

$$
D_{\varphi}(\mathscr{L})=\left\{u \in B / \int_{0}^{1} x^{k} u(x, t) d x=0, k=\overline{0,2 m-1} ; \ell u=\varphi\right\} .
$$

Theorem 3.1. Assume the function $a(\cdot, t) \in C^{2 m}([0,1])$ satisfies the conditions

$$
0<c_{k} \leq A_{m}^{k} \frac{\partial^{2 k} a}{\partial x^{2 k}}, \quad k=\overline{0, m}
$$

where $\left\{c_{k}, k=\overline{0, m}\right\}$ are real constants ( $A_{m}^{k}$ is defined by (2.8)). Then there exists a positive constant $c$ not depending on $u$ such that

$$
\|u\|_{B} \leq c\|\mathscr{L} u\|_{H}
$$

for all $u$ in $D_{\varphi}(\mathscr{L})$.

Proof. We take the scalar product in space $B_{2}^{m}(0,1)$ of equation (1.1) by multiplier $2 u$, that is

$$
2 \int_{0}^{1} \partial_{0 t}^{\alpha}\left(\Im_{x}^{m} u\right) \Im_{x}^{m} u d x+2(-1)^{m} \int_{0}^{1} a \frac{\partial^{m} u}{\partial x^{m}} \Im_{x}^{m} u d x=2 \int_{0}^{1} \Im_{x}^{m} f \Im_{x}^{m} u d x .
$$

Using lemma 2.1 on the first term of left-hand side of equation (3.3) we obtain

$$
2 \int_{0}^{1} \partial_{0 t}^{\alpha}\left(\Im_{x}^{m} u\right) \Im_{x}^{m} u d x \geq \int_{0}^{1} \partial_{0 t}^{\alpha}\left(\Im_{x}^{m} u\right)^{2} d x
$$


the second term in the left-hand side is estimated from proposition 2.3 and assumptions (3.1) as follows

$$
\begin{aligned}
2(-1)^{m} \int_{0}^{1} a \frac{\partial^{m} u}{\partial x^{m}} \Im_{x}^{m} u d x & =\sum_{k=0}^{m} A_{m}^{k} \int_{0}^{1} \frac{\partial^{2 k} a}{\partial x^{2 k}}\left(\Im_{x}^{k} u\right)^{2} d x \\
& \geq \sum_{k=0}^{m} c_{k} \int_{0}^{1}\left(\Im_{x}^{k} u\right)^{2} d x,
\end{aligned}
$$

for the right-hand side term of equation (3.3), Cauchy inequality (2.5) with $\varepsilon=c_{m}+1$ gives

$$
2 \int_{0}^{1} \Im_{x}^{m} f \Im_{x}^{m} u d x \leq\left(c_{m}+1\right) \int_{0}^{1}\left(\Im_{x}^{m} u\right)^{2} d x+\frac{1}{\left(c_{m}+1\right)} \int_{0}^{1}\left(\Im_{x}^{m} f\right)^{2} d x
$$

In light of inequalities (3.4),(3.5), and (3.6), one can get from (3.3)

$$
\int_{0}^{1} \partial_{0 t}^{\alpha}\left(\Im_{x}^{m} u\right)^{2} d x+\sum_{k=0}^{m-1} c_{k} \int_{0}^{1}\left(\Im_{x}^{k} u\right)^{2} d x \leq \int_{0}^{1}\left(\Im_{x}^{m} u\right)^{2} d x+\frac{1}{\left(c_{m}+1\right)} \int_{0}^{1}\left(\Im_{x}^{m} f\right)^{2} d x
$$

dropping the positive terms

$$
\sum_{k=0}^{m-1} c_{k} \int_{0}^{1}\left(\Im_{x}^{k} u\right)^{2} d x
$$

in inequality (3.7), replacing $t$ by $\tau$ and integrating with respect to $\tau$ from 0 to $t$ leads to

$$
\int_{0}^{t} \int_{0}^{1} \partial_{0 \tau}^{\alpha}\left(\Im_{x}^{m} u\right)^{2} d x d \tau \leq \int_{0}^{t} \int_{0}^{1}\left(\Im_{x}^{m} u\right)^{2} d x d \tau+\int_{0}^{t} \int_{0}^{1}\left(\Im_{x}^{m} f\right)^{2} d x d \tau
$$

and by setting

$$
y(t)=\int_{0}^{t} \int_{0}^{1}\left(\Im_{x}^{m} u\right)^{2} d x d \tau
$$

inequality (3.8) through lemma 2.2 induces

$$
y(t) \leq \Gamma(\alpha) E_{\alpha, \alpha}\left(t^{\alpha}\right) I^{\alpha+1} \int_{0}^{1}\left(\Im_{x}^{m} f\right)^{2} d x
$$

Also we have

$$
I^{\alpha+1} \int_{0}^{1}\left(\Im_{x}^{m} f\right)^{2} d x \leq \frac{T^{\alpha}}{\Gamma(\alpha+1)}\|f\|_{L^{2}\left(B_{2}^{m}(0,1),(0, T)\right)}^{2}
$$


and

$$
\int_{0}^{t} \int_{0}^{1} \partial_{0 \tau}^{\alpha}\left(\Im_{x}^{m} u\right)^{2} d x d \tau=I^{1-\alpha}\|u\|_{B_{2}^{m}(0,1)}^{2}-\frac{t^{1-\alpha}}{\Gamma(2-\alpha)}\|\varphi\|_{B_{2}^{m}(0,1)}^{2} .
$$

From inequalities (3.9), (3.10) and (3.11), inequality (3.7) using corollary 2.1 becomes

$$
\begin{gathered}
I^{1-\alpha}\|u\|_{B_{2}^{m}(0,1)}^{2}+\sum_{k=0}^{m-1} c_{k} \int_{0}^{t}\|u\|_{B_{2}^{k}(0,1)}^{2} d \tau \\
\leq \gamma\left(\|f\|_{L^{2}(Q)}^{2}+\|\varphi\|_{L^{2}(0,1)}^{2}\right)
\end{gathered}
$$

where

$$
\gamma=\frac{1}{2^{m}} \max \left\{1+\frac{E_{\alpha, \alpha}\left(T^{\alpha}\right) T^{\alpha}}{\alpha}, \frac{T^{1-\alpha}}{\Gamma(2-\alpha)}\right\} .
$$

Since the right hand side of the above inequality does not depend ont $t$, we can take the upper bound with respect to $t$ over $[0, T]$ to get the a priori estimate (3.2) with

$$
c=\left(\frac{\gamma}{\min _{0 \leq k<m}\left\{1, c_{k}\right\}}\right)^{\frac{1}{2}} \text {. }
$$

Proposition 3.1. The operator $\mathscr{L}$ from $B$ to $H$ has a closure $\overline{\mathscr{L}}$.

Proof. See proposition 10 in [6].

Consequently, the a priori estimate (3.2) can be extended to cover strong solutions by passing to the limit.

Corollary 3.1. Under assumptions (3.1) there exists a positive constant $c$ such that

$$
\|u\|_{B} \leq c\|\overline{\mathscr{L}} u\|_{H}
$$

for all $u$ in $D_{\varphi}(\overline{\mathscr{L}})$.

The uniqueness and continuous dependence of the solution on the problem data is now guaranteed in case of existence.

\section{Existence of the solution}

We aim to show the range density of operator $\mathscr{L}$ in Hilbert space $H$, that is $\overline{R(\mathscr{L})}=H$. We start by the case $u$ belongs to $D_{0}(\mathscr{L})$ (i.e. $\ell u=0$ ), after which follows the density in the general case $u \in D_{\varphi}(\mathscr{L})$ taking into consideration the fact that operator $\ell$ is everywhere dense.

Theorem 4.1. Assume for all $u$ in $D_{0}(\mathscr{L})$

$$
(\mathcal{L} u, \psi)_{L^{2}(Q)}=0,
$$

then under assumptions (3.1) $\psi$ vanishes a.e in $L^{2}(Q)$. 
Proof. Let us assume that a function $\theta(x, t)$ satisfies integral conditions (1.3) such that $\theta \in$ $B_{2}^{k}(0,1)$ and $\frac{\partial^{k} \theta}{\partial x^{k}} \in L^{2}(0,1), k=\overline{0, m}$. Then we can set

$$
u(x, t)=\int_{0}^{t} \theta(x, \tau) d \tau
$$

so that assumption (4.1) becomes

$$
\begin{gathered}
\int_{Q} \partial_{0 t}^{\alpha}\left(\int_{0}^{t} \theta(x, \tau) d \tau\right) \psi(x, t) d x d t \\
+(-1)^{m} \int_{Q} \frac{\partial^{m}}{\partial x^{m}}\left(a(x, t) \frac{\partial^{m}}{\partial x^{m}} \int_{0}^{t} \theta(x, \tau) d \tau\right) \psi(x, t) d x d t=0 .
\end{gathered}
$$

We now express $\psi$ in terms of $\theta$ :

$$
\psi(x, t)=2(-1)^{m} \int_{0}^{t} \Im_{x}^{2 m} \theta(x, \tau) d \tau
$$

to get from equation (4.2)

$$
\begin{gathered}
2 \int_{Q}\left(\int_{0}^{t} \Im_{x}^{m} \theta(x, \tau) d \tau\right) \partial_{0 t}^{\alpha}\left(\int_{0}^{t} \Im_{x}^{m} \theta(x, \tau) d \tau\right) d x d t \\
+2(-1)^{m} \int_{Q} a(x, t)\left(\int_{0}^{t} \frac{\partial^{m}}{\partial x^{m}} \theta(x, \tau) d \tau\right)\left(\int_{0}^{t} \Im_{x}^{m} \theta(x, \tau) d \tau\right) d x d t=0 .
\end{gathered}
$$

From lemma 2.1 we have

$$
2\left(\int_{0}^{t} \Im_{x}^{m} \theta(x, \tau) d \tau\right) \partial_{0 t}^{\alpha}\left(\int_{0}^{t} \Im_{x}^{m} \theta(x, \tau) d \tau\right) \geq \partial_{0 t}^{\alpha}\left(\int_{0}^{t} \Im_{x}^{m} \theta(x, \tau) d \tau\right)^{2},
$$

and using proposition 2.3 one can get

$$
\begin{gathered}
2(-1)^{m} \int_{0}^{1} a(x, t)\left(\int_{0}^{t} \frac{\partial^{m}}{\partial x^{m}} \theta(x, \tau) d \tau\right)\left(\int_{0}^{t} \Im_{x}^{m} \theta(x, \tau) d \tau\right) d x= \\
\sum_{k=0}^{m} A_{m}^{k} \int_{0}^{1} \frac{\partial^{2 k} a}{\partial x^{2 k}}\left(\int_{0}^{t} \Im_{x}^{k} \theta(x, \tau) d \tau\right)^{2} d x .
\end{gathered}
$$


Hence by using assumptions (3.1) and substituting the last two relations in equation (4.3) we deduce

$$
\int_{Q} \partial_{0 t}^{\alpha}\left(\int_{0}^{t} \Im_{x}^{m} \theta(x, \tau) d \tau\right)^{2} d x d t+\sum_{k=0}^{m} c_{k} \int_{Q}\left(\int_{0}^{t} \Im_{x}^{k} \theta(x, \tau) d \tau\right)^{2} d x d t \leq 0,
$$

by taking the upper bound ovet $[0, T]$ for both sides of the above inequality yields

$$
\sup _{0 \leq t \leq T} I^{1-\alpha}\left\|\int_{0}^{t} \Im_{x}^{m} \theta(x, \tau) d \tau\right\|_{L^{2}(0,1)}^{2}+\sum_{k=0}^{m} c_{k}\left\|\int_{0}^{t} \Im_{x}^{k} \theta(x, \tau) d \tau\right\|_{L^{2}(Q)}^{2} \leq 0 .
$$

Consequently, $\theta=0$ and $\psi=0$ a.e in $L^{2}(Q)$.

Now consider the general case. Let $\mathscr{L}_{0}$ denotes the operator $\left(\mathcal{L}_{0}, 0\right)$, If we use the fact that $\mathscr{L}-\mathscr{L}_{0}=\left(\mathcal{L}-\mathcal{L}_{0}, \ell\right)$ maps continuously $B$ into $H$, we conclude that $R(\mathscr{L})$ is dense in $H$ by means of the method of continuation along the parameter (see [3]).

Example 4.1. Let $\beta=7(189 \ln 2-131), \gamma=(1416-2043 \ln 2), \delta=\frac{15}{2}(114 \ln 2-79), \mu=$ $\frac{1}{10}(535-774 \ln 2)$ and

In the problem (1.1)-(1.3) we take for $m=2$

$$
\omega(x)=7 x^{4}-14 x^{3}+9 x^{2}-2 x+\frac{1}{10} .
$$

$$
\begin{gathered}
a(x, t)=t-\frac{x^{2}}{2}+1 \\
\varphi(x)=\beta x^{3}+\gamma x^{2}+\delta x+\mu+\frac{3}{20(x+1)},
\end{gathered}
$$

and

$f(x, t)=\frac{\omega(x)}{\Gamma(2-\alpha)} t^{1-\alpha}-504 t x^{2}+18(14 t-\beta) x+2\left(84 t^{2}+75 t-\gamma\right)+\frac{3\left(12 t-x^{2}+4 x+11\right)}{10(x+1)^{5}}$.

Obviously, $f \in L^{2}(Q)$ and the function a satisfies assumptions of theorem (3.1). We conclude that problem (1.1)-(1.3) admits a unique solution for the given data. In addition we can show by an elementary calculation that

$$
u(x, t)=t \omega(x)+\varphi(x)
$$

fulfills equation (1.1), initial condition (1.2) and integral conditions (1.3), therefore it is the desired unique solution.

Remark 4.1. Solvability of the problem obtained by replacing operator $\mathcal{L}$ with

$$
\mathcal{L} u \equiv \partial_{0 t}^{\alpha} u+(-1)^{m} \frac{\partial^{m}}{\partial x^{m}}\left(a(x, t) \frac{\partial^{m} u}{\partial x^{m}}\right)+b(x, t) u
$$

can be established in the same way assuming the boundness of function $b$. 


\section{Conclusion}

In this paper, the existence and uniqueness of a Caputo time fractional problem with boundary integral conditions are established. we applied the "energy inequality" method, which is a traditional functional analysis method. Among the tools used to work out the solvability of the problem, a generalized formula of integration was demonstrated and applied, thus the outcoming results will contribute in the development of the functional analysis methods whether in time fractional case or integer-order case. In addition of the problem in the remark above, we are currently investigating several time fractional problems with both Caputo and Riemann derivatives including a numerical study.

\section{Acknowledgments}

The authors thank the anonymous referees for their valuable suggestions and comments which allowed to correct and improve the paper.

\section{Competing interests}

The authors declare that they have no competing interests.

\section{Authors contributions}

Both authors discussed the results and contributed to the final manuscript.

\section{References}

[1] A.A. Alikhanov, A priori estimates for solutions of boundary value problems for fractional-order equations, Differential Equations, Vol. 46, No. 5, pp. 660-666, 2010. https://doi.org/10.1134/S0012266110050058

[2] A. Bouziani, Mixed problem with boundary integral conditions for a certain parabolic equation, Journal of Applied Mathematics and Stochastic Analysis, vol. 9, no. 3, pp. 323-330, 1996. https://doi.org/10.1155/ S1048953396000305

[3] A. Bouziani,Initial boundary value problem with a non-local condition for a viscosity equation, Hindawi Publishing Corp, Vol 30, No.6, pp. 327-338, 2002. https://doi .org/10.1155/S0161171202004167

[4] A.L. Marhoune and C. Latrous, A strong solution of a high-order mixed type partial differential equation with integral conditions, Applicable Analysis,Vol. 87, No. 6, pp. 625-634, 2008.

[5] A. Merad, A.L. Marhoune, Strong solution for a high order boundary value problem with integral condition, Turkish Journal of Mathematics 37 (2), 299-307, 2013. https://doi .org/10 .3906/mat-1105-34

[6] A. Merad, J. Martin-Vaquero, A Galerkin method for two-dimensional hyperbolic integro-differential equation with purely integral conditions, Applied Mathematics and Computation, Vol 291, pp. 386-394, 2016. https: //doi.org/10.1016/j.amc.2016.07.003

[7] I. Podlubny, Fractional Differential Equations, Academic Press, San Diego, 1999.

[8] J. Martin-Vaqueroa, A. Merad, Existence, uniqueness and numerical solution of a fractional PDE with integral conditions, Nonlinear Analysis: Modelling and Control, Vol. 24, No. 3, pp. 368-386, 2019. https://doi.org/ 10.15388/NA.2019.3.4 